\title{
Extensive Sex Chromosome Polymorphism of Microtus thomasi/Microtus atticus Species Complex Associated with Cryptic Chromosomal Rearrangements and Independent Accumulation of Heterochromatin
}

\author{
Michail T. Rovatsos ${ }^{a}$ Juan A. Marchal ${ }^{b}$ Ismael Romero-Fernández ${ }^{b}$ \\ Maria Arroyo $^{\text {b }}$ Eva B. Athanasopoulouc Antonio Sánchez ${ }^{b}$ \\ ${ }^{a}$ Department of Ecology, Faculty of Science, Charles University in Prague, Praha, Czech Republic; ${ }^{b}$ Departamento \\ de Biología Experimental, Facultad de Ciencias Experimentales, Universidad de Jaén, Campus Las Lagunillas, Jaén, \\ Spain; ' Section of Animal Biology, Department of Biology, University of Patras, Rion, Greece
}

\section{Keywords}

Chromosome painting $\cdot$ Microtus $\cdot$ Polymorphism $\cdot$ Sex chromosome heterochromatin

\begin{abstract}
The sibling species Microtus thomasi and M. atticus represent probably the highest karyotypic diversity within the genus Microtus and are an interesting model for chromosomal evolution studies. In addition to variation in autosomes, they show a high intraspecific variation in the size and morphology of both sex chromosomes. We analyzed individuals with different sex chromosome constitutions using 3 painting probes, 2 from $Y$ chromosome variants and 1 from the small arm of the submetacentric $X$ chromosome. Our comparative painting approach uncovered 12 variants of $Y$ and 14 variants of X chromosomes, which demonstrates that the polymorphism of sex chromosomes is substantially larger than previously reported. We suggest that 2 main processes are responsible for this sex chromosome polymorphism: change of morphology from acrocentric to submetacentric or metacentric chromosomes and increase in size due to accumulation of repetitive DNA sequences, generating heterochro-
\end{abstract}

\section{KARGER}

(C) 2017 S. Karger AG, Basel

E-Mail karger@karger.com

www.karger.com/cgr matic blocks. Strong genetic drift in small and fragmented populations of these 2 species could be related to the origin and maintenance of the large polymorphism of sex chromosomes. We proposed that a similar polymorphism variation combined with random drift fixing the biggest sex chromosomes could have occurred in the origin of some of the actual Microtus species with giant sex chromosomes.

(c) 2017 S. Karger AG, Basel

The rodent species of the subfamily Arvicolinae constitute one of the most variable mammalian groups characterized by high karyotypic variation, especially in the genus Microtus that is associated with rapid speciation events [Conroy and Cook, 1999; Musser and Carleton, 2005]. This genus includes 65 extant species currently classified into 14 subgenera [Musser and Carleton, 2005; Golenishchev and Malikov, 2006] and presents the most remarkable rate of karyotypic variability and evolution among rodents [Maruyama and Imai, 1981; Lemskaya et al., 2010]. The chromosomal variation ranges from $2 \mathrm{n}=$ 18 in Microtus oregoni to 2n =64 in M. longicaudus [Modi, 1987a; Volobouev et al., 2006]. In relation to the karyo-

Antonio Sánchez

Departamento de Biología Experimental, Facultad de Ciencias Experimentales Universidad de Jaén, Paraje Las Lagunillas $s / n$

ES-23071 Jaén (Spain)

E-Mail abaca@ujaen.es 
Table 1. Additional individuals of $M$. thomasi analyzed with indication of the locality of trapping, the specimen code, the chromosomal race, the chromosome number (2n), the fundamental number $(\mathrm{FN})$, sex, and the variants of sex chromosomes

\begin{tabular}{|c|c|c|c|c|c|c|c|}
\hline Locality & Specimen & Chromosomal race ${ }^{\mathrm{a}}$ & $2 n$ & FN & Sex & $\mathrm{X}-\mathrm{chr}$ & Y-chr \\
\hline Mpalntouma, Greece & D20 & hybrid “thomasi”×"Rb subalpine” & 43 & 44 & female & $\mathrm{X}_{\mathrm{st1} 1}-\mathrm{X}_{0}$ & \\
\hline Mpalntouma, Greece & 7889 & hybrid "Kastrosikia"×"Rb subalpine" & 41 & 44 & male & $\mathrm{X}_{\mathrm{st1}}$ & $\mathrm{Y}_{1}$ \\
\hline Kali, Greece & $\mathrm{R} 27$ & "Kali" & 40 & 42 & female & $\mathrm{X}_{3}-\mathrm{X}_{3}$ & \\
\hline Aridea, Greece & $\mathrm{R} 152$ & "Aridea" & 38 & 42 & male & $\mathrm{X}_{4}$ & $\mathrm{Y}_{2}$ \\
\hline Veria, Greece & R252 & "subalpine" & 42 & 42 & male & $\mathrm{X}_{4}$ & $\mathrm{Y}_{1}$ \\
\hline Ducat, Albania & R160 & "subalpine" & 42 & 42 & female & $\mathrm{X}_{4}-\mathrm{X}_{4}$ & \\
\hline
\end{tabular}

Data about the rest of individual analyzed is shown in Rovatsos et al. [2014]. a According to Rovatsos and Giagia-Athanasopoulou [2012].

typic variation, the sex chromosomes are also highly variable in morphology, ranging from an acrocentric to a metacentric shape, and in size due to accumulation of constitutive heterochromatin in both $\mathrm{X}$ and $\mathrm{Y}$ chromosomes [Modi, 1987a, b; Burgos et al., 1988a, b; Mitsainas et al., 2009; Marchal et al., 2003, 2004a; Acosta et al., 2011]. In some cases, the heterochromatin accumulation led to a notable increase in size of both sex chromosomes, which represent a significant part of the genome and are referred to as 'giant' sex chromosomes. Such giant sex chromosomes occur in the species M. agrestis, M. cabrerae, M. chrotorrhinus, M. rossiaemeridionalis, and $M$. transcaspicus [Modi, 1987a, b; Nanda et al., 1988; Kalscheuer et al., 1996].

The sibling voles $M$. thomasi and M. atticus are interesting models for studies on chromosomal evolution as they demonstrate probably the highest rate of karyotypic diversity among the Microtus species. Nine chromosomal races in $M$. thomasi and 2 in $M$. atticus were described [Giagia-Athanasopoulou and Stamatopoulos, 1997; Mitsainas et al., 2009; Rovatsos et al., 2011a, 2014; Rovatsos and Giagia-Athanasopoulou, 2012]. The karyotype of the chromosomal race assigned as $M$. thomasi 'thomasi' with all chromosomes being acrocentric $(2 \mathrm{n}=44, \mathrm{FN}=44)$ was considered to represent a likely ancestral karyotype for this species complex [Giagia-Athanasopoulou and Stamatopoulos, 1997]. The variation in chromosome number among chromosomal races can be probably attributed to Robertsonian and tandem fusions [Giagia-Athanasopoulou et al., 1995; Giagia and Ondrias, 1973; Giagia, 1985; Rovatsos et al., 2011a, 2014; Rovatsos and Giagia-Athanasopoulou, 2012].

In addition to variation in autosomes, $M$. thomasi and M. atticus show a high intraspecific variation in size and morphology of both sex chromosomes. C-banding tech-

Sex Chromosome Polymorphism of M. thomasi/M. atticus nique demonstrated a significant variation in the amount of pericentromeric heterochromatin and in the size and position of heterochromatic bands or blocks. Nine different $\mathrm{X}$ chromosomes have been described with either acrocentric $\left(\mathrm{X}_{0}-\mathrm{X}_{5}\right)$ or subtelocentric $\left(\mathrm{X}_{\mathrm{st0}}-\mathrm{X}_{\mathrm{st} 2}\right)$ morphology and variable amounts of heterochromatin [Acosta et al., 2009; Mitsainas et al., 2009; Rovatsos et al., 2011a, 2014]. Similarly, 8 Y chromosome variants exist, either acrocentric $\left(\mathrm{Y}_{0}-\mathrm{Y}_{4}\right)$ or metacentric $\left(\mathrm{Y}_{\mathrm{m}}\right.$ and $\left.2 \mathrm{Y}_{\mathrm{m} 1}\right)$; all of them are almost completely heterochromatic and also highly variable in size [Acosta et al., 2009; Mitsainas et al., 2009; Rovatsos et al., 2011a; Rovatsos and Giagia-Athanasopoulou, 2012].

Several studies have shed light on the molecular composition and the evolution of sex chromosomes in arvicolids. These investigations have characterized different families of complex repeated DNAs, satellite DNAs, retroelements, and pseudogenes as components of the sex chromosome heterochromatin [Marchal et al., 2003, 2004b, 2006, 2008; Acosta et al., 2008, 2009; Rovatsos et al., 2014]. Many of these sequences are significantly accumulated in the heterochromatic blocks of the giant sex chromosomes and display patterns of distribution, organization, and amplification that are highly variable within species. Also, comparative painting with the giant $\mathrm{X}$ and $\mathrm{Y}$ chromosomes of $M$. cabrerae and M. agrestis revealed the rapid and independent evolution of the sequences forming the heterochromatic blocks and the conservation of the euchromatic X content [Marchal et al., 2004a; Sitnikova et al., 2007].

In particular, 4 repetitive DNA sequences located on the pericentromeric chromosomal regions have been characterized in M. thomasi: Msat-160 [Acosta et al., 2010], Mth-Alu900 [Acosta et al., 2009], Mth-Alu2.2 [Rovatsos et al., 2014], and interstitial telomeric sequences

Cytogenet Genome Res 2017;151:198-207 DOI: $10.1159 / 000477114$ 

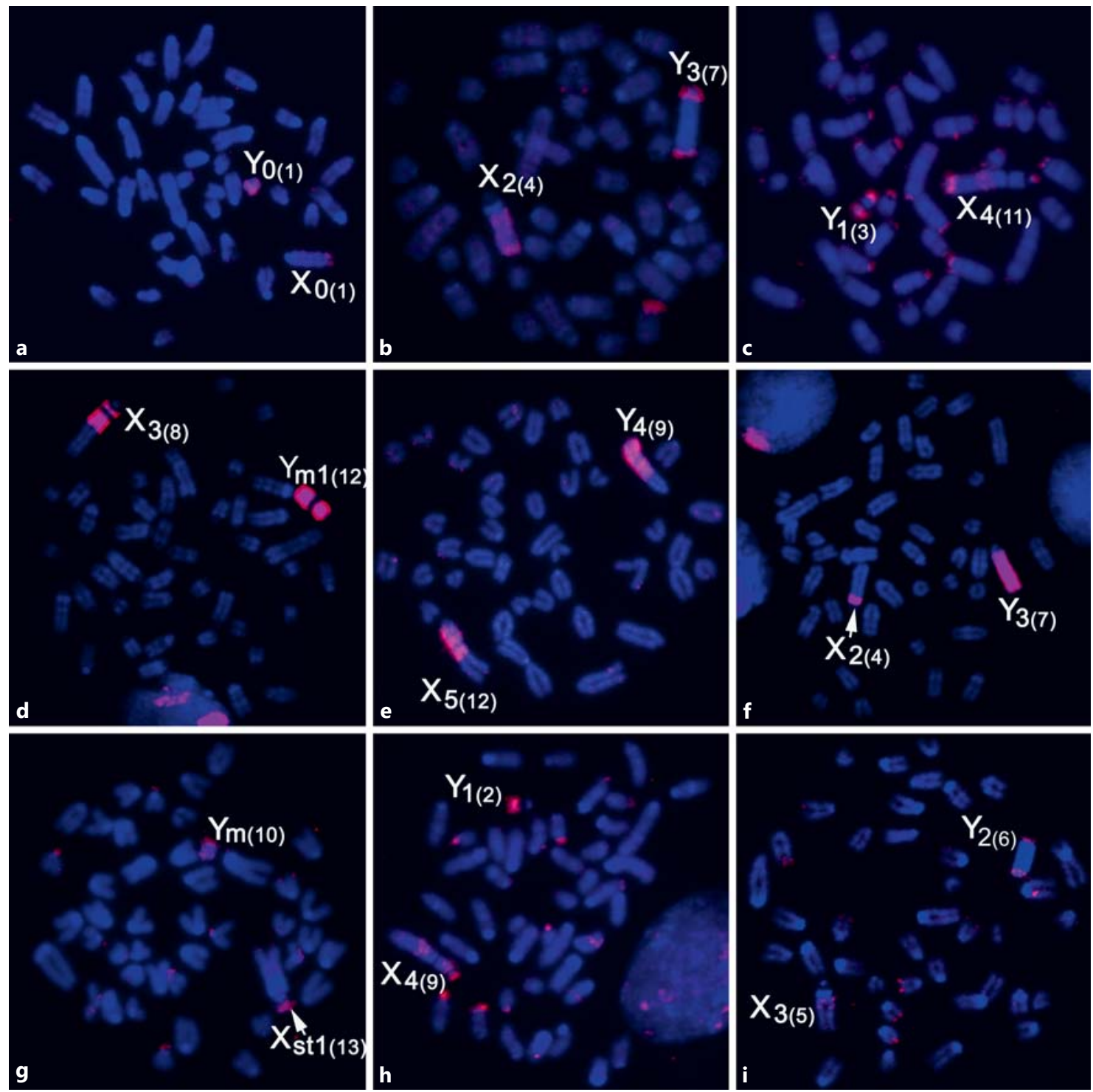

Fig. 1. Examples of painting on chromosomes of different individuals with the painting probes $\mathrm{Y}_{0}(\mathbf{a}-\mathbf{c}), \mathrm{Y}_{\mathrm{m} 1}$ $(\mathbf{d}-\mathbf{f})$, and $\mathrm{X}_{\mathrm{st1}} \mathrm{p}(\mathbf{g}-\mathbf{i})$. Notation of sex chromosomes includes the classification of C-banding $\mathrm{X}_{\#}$ or $\mathrm{Y}_{\#}$ according to Rovatsos et al. [2014], and in brackets the variant number of Figures 2 and 3 identified by painting are shown.

[Rovatsos et al., 2011b]. In sex chromosomes, the highly repetitive satellite sequence Mth-Alu900 was located in the pericentromeric heterochromatin but not in the proximal or interstitial C-bands of the acrocentric X chromosome in this species. However, this satellite DNA is located also in the submetacentric $\mathrm{X}_{\text {st1 }}$ chromosome in the adjacent heterochromatic blocks. Only the large, completely heterochromatic $\mathrm{Y}_{2}$ chromosome variant presents this satellite DNA in its pericentromeric heterochromatin [Acosta et al., 2009]. In addition, the Mth-Alu2.2 is located in the heterochromatic block of $\mathrm{X}_{\mathrm{st1}}$ and in the ter- minal or interstitial small bands in the other X or Y chromosome variants [Rovatsos et al., 2014]. The telomericlike repetitive sequences are located in the heterochromatin of $2 \mathrm{X}$ chromosome variants analyzed by Rovatsos et al. [2011b] as well.

The sex chromosome system in M. thomasi/M. atticus has been studied extensively only by C-banding. The location of Mth-Alu900 and Mth-Alu2.2 repetitive sequences in sex chromosomes suggested that the polymorphism detected by classical cytogenetic techniques could be even more pronounced at the molecular level [Acosta 
Fig. 2. Representative Y chromosome variants showing the painting pattern with the painting probes $\mathrm{Y}_{0}, \mathrm{Y}_{\mathrm{ml}}$, and $\mathrm{X}_{\mathrm{st} 1 \mathrm{p}} \mathrm{p}$, and the C-banding. Notation of sex chromosomes includes the order number according to painting identification ( $\mathrm{pY} \#)$ and the classification with $\mathrm{C}$-banding $\mathrm{Y}_{\#}$ according to Rovatsos et al. [2014].

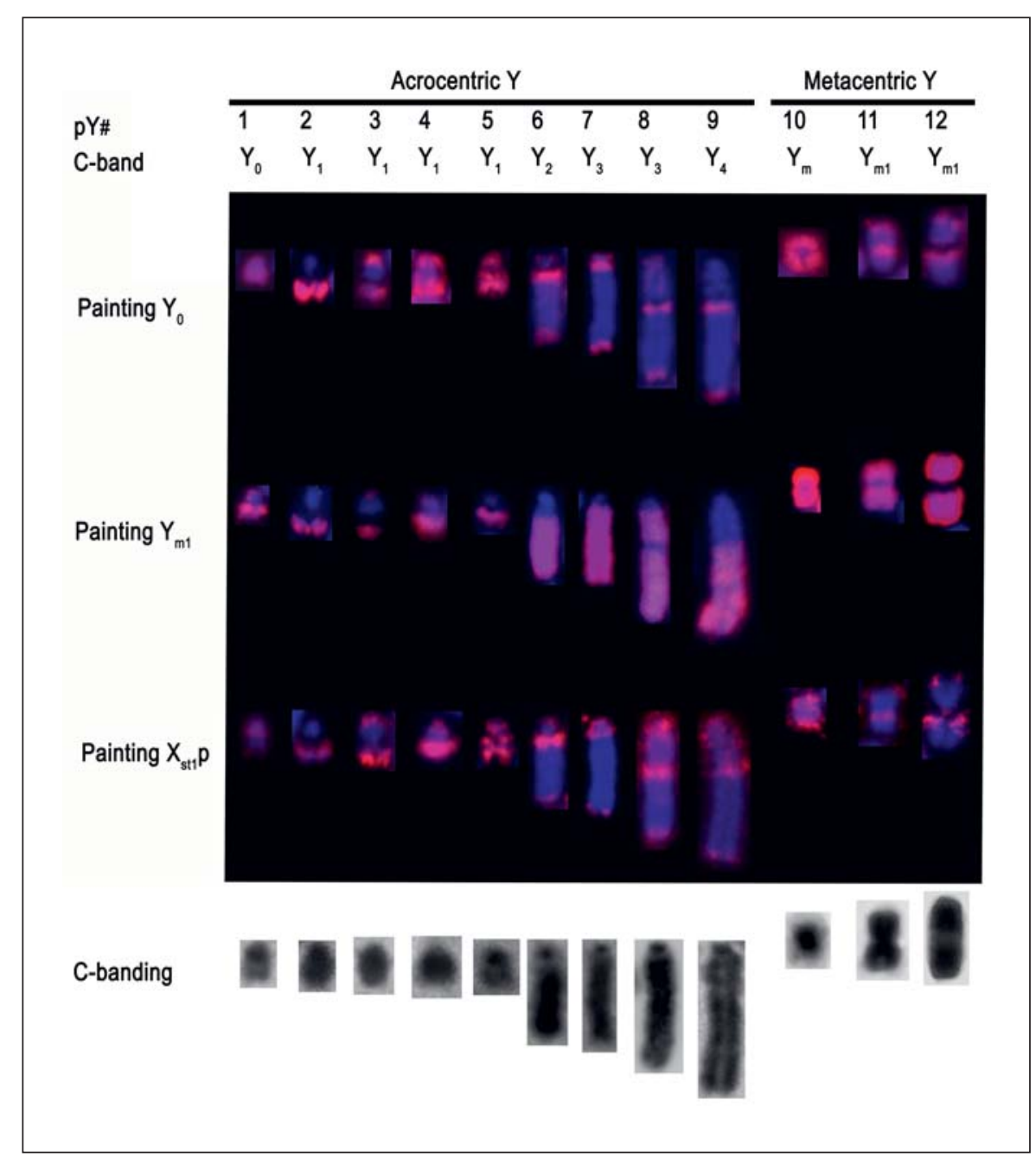

et al., 2009; Rovatsos et al., 2014]. A detailed analysis by molecular cytogenetics was required in order to better characterize this polymorphism. Here, we present the analysis of most of the sex chromosome variants described in $M$. thomasi/M. atticus based on comparative chromosome painting with 3 probes derived from $2 \mathrm{Y}$ chromosome variants $\left(\mathrm{Y}_{0}\right.$ and $\left.\mathrm{Y}_{\mathrm{m} 1}\right)$ and the small arm of the submetacentric $\mathrm{X}_{\mathrm{st} 1}$ chromosome.

\section{Material and Methods}

\section{Individuals Analyzed and Chromosome Preparations}

For this study, we used chromosome preparations from 26 individuals of different chromosomal races of 2 species from the subgenus Terricola of the genus Microtus: M. thomasi and M. atticus belonging to different chromosomal races from Greece, Albania, and Montenegro. The data about most of the specimens utilized in this work is shown in Rovatsos et al. [2014]. In addition, we included additional 6 specimens of different chromosomal races of the species $M$. thomasi (Table 1).

Chromosomes were prepared from bone marrow using a modified version of the Hsu and Patton [1969] method. The karyotypes of the individuals have different sex chromosome constitutions and were previously analyzed by C-banding [Mitsainas et al., 2009; Rovatsos et al., 2011a].

\section{Chromosome Painting}

Three painting probes were prepared from the whole small acrocentric $\mathrm{Y}_{0}$ chromosome (M. thomasi specimen R32), the biggest metacentric $\mathrm{Y}_{\mathrm{ml}}$ chromosome (M. thomasi specimen R94), and the small heterochromatic $\mathrm{p}$ arm of the submetacentric $\mathrm{X}_{\mathrm{stl}}$ chromosome (specimen R32). For probe preparation and painting, we followed the protocol previously described by our group [Marchal et al., 2004a]. Briefly, microdissection was performed using an inverted microscope (Zeiss Axiovert S100) with a sterile glass needle attached to a mechanical micromanipulator (Eppendorf TransferMan NK2). A total of 10-15 microdissected chromosomes were 
Fig. 3. Representative X chromosome variants showing the painting pattern with the painting probes $\mathrm{Y}_{0}, \mathrm{Y}_{\mathrm{m} 1}$, and $\mathrm{X}_{\mathrm{st} 1 \mathrm{p}} \mathrm{p}$, and the C-banding. Notation of sex chromosomes includes the order number according to painting identification ( $\mathrm{pX \# )}$ and the classification with $\mathrm{C}$-banding $\mathrm{X}_{\#}$ according to Rovatsos et al. [2014].

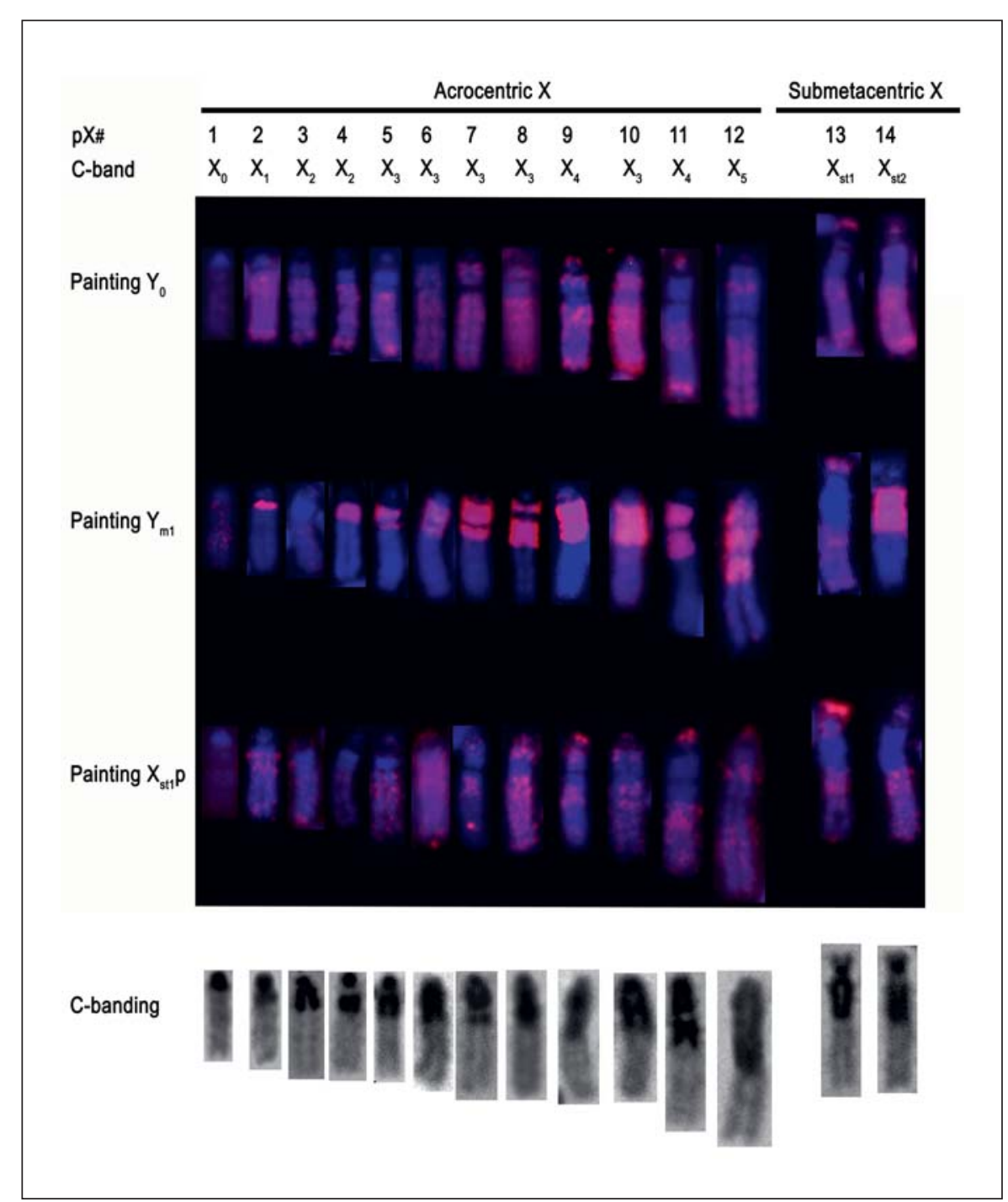

used in an initial DOP-PCR amplification, and a second labelling DOP-PCR using Spectrum-Orange dUTP (Abbott) was performed to obtain the painting probe. Approximately $200 \mathrm{ng}$ of probe were used for every FISH analysis. The images were captured using a fluorescence microscope (Olympus BX51) equipped with a CCD camera (Olympus DP70).

\section{Results}

The 3 painting probes $\left(\mathrm{Y}_{0}, \mathrm{Y}_{\mathrm{m} 1}\right.$, and $\left.\mathrm{X}_{\mathrm{st} 1} \mathrm{p}\right)$ hybridized as expected to the entire corresponding chromosome or chromosome region from which they were prepared (Fig. 1a, d, g), but also to various parts of the Y chromosome in different $\mathrm{Y}$ variants, most of the $\mathrm{X}$ chromosome variants, and depending on each particular karyotype, a variable number of signals was observed in autosomes either in pericentromeric heterochromatin and in interstitial euchromatic bands (Fig. 1-3). Based on the size, Cbanding, and the current painting results, we were able to identify $12 \mathrm{Y}$ chromosome variants (5 small acrocentric and 4 big acrocentric and 3 metacentric chromosomes of variable size) (Fig. 2) and $14 \mathrm{X}$ chromosome variants (12 acrocentrics with variable size and 2 submetacentric chromosomes) (Fig. 3). Below we refer to the different variants of the $\mathrm{X}$ and $\mathrm{Y}$ chromosomes identified by painting as pX\# and pY\#, were \# is the order number of each chromosome in Figures 2 and 3, while we refer to the corresponding variant described by $\mathrm{C}$-banding with the previously used notation ( $\mathrm{X}$ chromosomes: $\mathrm{X}_{0}-\mathrm{X}_{5}, \mathrm{X}_{\mathrm{st} 0}-\mathrm{X}_{\mathrm{st} 2}$; $\mathrm{Y}$ chromosomes: $\mathrm{Y}_{0}-\mathrm{Y}_{4}, \mathrm{Y}_{\mathrm{m}}$ and $\left.\mathrm{Y}_{\mathrm{m} 1}\right)$. 
Painting with the $Y_{0}$ Probe

As expected, this probe hybridized to the complete $\mathrm{Y}_{0}$ chromosome (pY1) (Fig. 1a) but gave different signals on the other Y chromosome variants (Fig. 1a-c, Fig. 2). In the 5 small acrocentric Y chromosomes (pY1-pY5), the staining occupies the entire chromosome or 1 or 2 terminal bands. In the 4 large acrocentric Y chromosomes (pY6-pY9), the signal was located to 2 main bands, 1 of them always in the telomeric region of the long arm, and the other close to the centromere in the long arm or in the pericentromeric region of the small (pY6 and pY7) or as an interstitial band in the long arm in the big chromosomes (pY8 and pY9). In the metacentric Y chromosomes this probe stained the entire small Y metacentric chromosome (pY10), while in the 2 big metacentrics (pY11 and pY12) only 2 bands were stained, 1 in the telomeric region of the small arm and another in the pericentromeric region (Fig. 2).

The $\mathrm{Y}_{0}$ probe also hybridized to most of the $\mathrm{X}$ chromosome variants with the exception of the small one (pX1) (Fig. 1a-c, Fig. 3). In the acrocentric X chromosomes (pX2-pX12), the signal was diverse in its intensity in the entire euchromatic region, and in some of them the probe also hybridized to some heterochromatic bands and pericentromeric regions (Fig. 3). In the same way, the probe hybridized to the entire euchromatic part of the long arm of the submetacentric X chromosomes (pX13 and pX14) but faintly to $\mathrm{X}_{\mathrm{st} 1}$ (pX13) and intensely to $\mathrm{X}_{\mathrm{st} 2}$ chromosomes (pX14). In addition, in both chromosomes it stained a terminal position in the heterochromatic small arm, which was big and more intense in Xst1 (pX13) (Fig. 3).

\section{Painting with the $Y_{m 1}$ Probe}

This probe hybridized as expected to the complete $\mathrm{Y}_{\mathrm{m} 1}$ chromosome (pY12) with the exception of the pericentromeric region (Fig. 1d) and also gave different signals in all Y chromosome variants (Fig. 1d-f, Fig. 2). In the 5 small acrocentric $\mathrm{Y}$ chromosomes (pY1-pY5), the probe stained almost entirely the variant $\mathrm{pY} 1$ and one intense band in the telomeric region of the long arm in the variants pY2-pY5. In addition, the variant pY3 showed a signal in the pericentromeric region. In the large acrocentric Y chromosomes (pY6-pY9) it stained almost the entire heterochromatic long arm with the exception of a region variable in size close to the centromeric region and of an interstitial band in one of them (pY8). In the metacentric Y chromosomes ( $\mathrm{pY} 10-\mathrm{pY} 12)$ the probe stained the entire chromosome with the exception of the pericentromeric region in the largest of them (pY12).

Sex Chromosome Polymorphism of M. thomasi/M. atticus
The $\mathrm{Y}_{\mathrm{m} 1}$ probe stained the heterochromatic block/ band of the acrocentric X chromosomes, with the exception of $\mathrm{pX} 3$. As this probe did not paint the pericentromeric heterochromatin, no signal was found in the variants pX1 that lacked heterochromatic bands (Fig. 3). Despite practically identical morphology, this probe permitted the clear differentiation of the 2 submetacentric $\mathrm{X}$ chromosomes $\left(\mathrm{X}_{\mathrm{st1}}\right.$ and $\left.\mathrm{X}_{\mathrm{st} 2}\right)$. In $\mathrm{X}_{\mathrm{st1}}(\mathrm{pX} 13)$ the probe faintly stained the euchromatic region of the long arm and a terminal band in the small heterochromatic arm, while in $\mathrm{X}_{\mathrm{st} 2}$ (pX14) it stained exclusively and intensely the heterochromatic block of the long arm proximal to the centromere (Fig. 3).

\section{Painting with the $X_{\text {st }} p$ Probe}

The pattern of painting with this probe was very similar to the pattern obtained with the $\mathrm{Y}_{0}$ probe in variants of both $\mathrm{X}$ and $\mathrm{Y}$ chromosomes (Fig. 1g-i, Fig. 2, Fig. 3). However, some of the euchromatic regions of the $\mathrm{X}$ chromosome variants showed less intense signals than with the $\mathrm{Y}_{0}$ probe (Fig. 3). Also, the large $\mathrm{Y}$ acrocentric chromosomes variants (pY8 and pY9) were stained with this probe between the interstitial band of the long arm and the centromere, which is mainly absent with the $\mathrm{Y}_{0}$ probe (Fig. 2). These results demonstrated that the $X_{\text {st1 }}$ short arm shared repetitive sequences with the $\mathrm{Y}_{0}$ chromosome responsible for the similar painting pattern, but in addition it contains also other repetitive sequences not located on the $\mathrm{Y}_{0}$ chromosome.

\section{Discussion}

For the first time we applied the chromosome painting technique to analyze the sex chromosome polymorphism in M. thomasi and M. atticus, where 9 variants of $\mathrm{X}$ chromosomes and 8 variants of $\mathrm{Y}$ chromosomes were previously described based on C-banding pattern, size, and morphology [Acosta et al., 2009; Mitsainas et al., 2009; Rovatsos et al., 2011a; Rovatsos and Giagia-Athanasopoulou, 2012; Rovatsos et al., 2014]. Our result demonstrated that this polymorphism is even more extensive than previously shown. On the basis of the painting pattern with the 3 chromosomal probes, we were able to differentiate $14 \mathrm{X}$ chromosome variants (pX1pX14) and 12 Y chromosomes variants (pY1-pY12) (Fig. 2, 3). Moreover, this polymorphism could be even larger, as for example the previously characterized submetacentric $\mathrm{X}_{\mathrm{st} 0}$ chromosome is not included in this study.

Cytogenet Genome Res 2017;151:198-207 DOI: $10.1159 / 000477114$ 


\section{Evolutive Dynamics of Y Chromosomes}

We recognized 5 small acrocentric, 4 large acrocentric, and 3 metacentric $Y$ chromosomes. All 5 small acrocentric variants were previously assigned as $\mathrm{Y}_{0}$ and $\mathrm{Y}_{1}$; however, we found 4 different $Y_{1}$ chromosomes (pY2-pY5) on the basis of the painting results. These chromosomes presented subtle variation in size, but the painting pattern suggests that they share some regions with similar repeated sequences, while other regions do not contain the same sequences. We assume that the smallest chromosome (pY1) is the ancestral one, and the other variants could be derived by amplification of different repeated DNA sequences in the heterochromatin. The 2 big acrocentric chromosomes (pY6 and pY7), assigned as $\mathrm{Y}_{2}$ and $\mathrm{Y}_{3}$, arose from a small acrocentric chromosome by a significant amplification of the heterochromatic block stained with the $\mathrm{Y}_{\mathrm{m} 1}$ probe, leaving the terminal regions of the long arm with sequences of the original $\mathrm{Y}$ small chromosome (these regions are stained by the $\mathrm{X}_{\mathrm{st} 1} \mathrm{p}$ and $\mathrm{Y}_{0}$ probes). The 2 largest acrocentric $\mathrm{Y}$ chromosomes (pY8 and pY9), assigned as $\mathrm{Y}_{3}$ and $\mathrm{Y}_{4}$, probably arose from one of the other big acrocentrics (pY6 or pY7) by acquisition of additional heterochromatin in the long arm proximal to the centromere. The acquired heterochromatic sequences in $\mathrm{pY} 8$ have the same composition than the rest of the arm and the $\mathrm{Y}_{\mathrm{m} 1}$ chromosomes, while in pY9 they have a different composition, although the heterochromatin in both chromosomes also shares some sequences stained with the $\mathrm{X}_{\mathrm{st1}} \mathrm{p}$ probe.

The small metacentric $Y$ chromosome (pY10) arose by a pericentric inversion or by accumulation of heterochromatic sequences on the small arm of some of the small acrocentric chromosomes, probably the variant pY4 or pY5. The other 2 big metacentric Y chromosomes (pY11 and pY12) could have arisen through heterochromatin amplification in both arms of the small metacentric $\mathrm{Y}$ or by a pericentric inversion in one of the big acrocentric Ys, probably the variant pY6 or pY7. The biggest one (pY12) additionally contains some different repetitive DNA sequences in the pericentromeric region that are not stained with the $\mathrm{Y}_{\mathrm{m} 1}$ probe.

The proposed origins for the different $\mathrm{Y}$ chromosome variants are in agreement with the geographic location of each of them, which is represented in Figure 4.

\section{Evolutive Dynamics of X Chromosomes}

We found 12 acrocentric and 2 submetacentric $X$ chromosomes. Giagia-Athanasopoulou and Stamatopoulos [1997] suggested that the ancestral X chromosome in $M$. thomasi is the acrocentric one with pericentromeric heterochromatin but without heterochromatic bands.

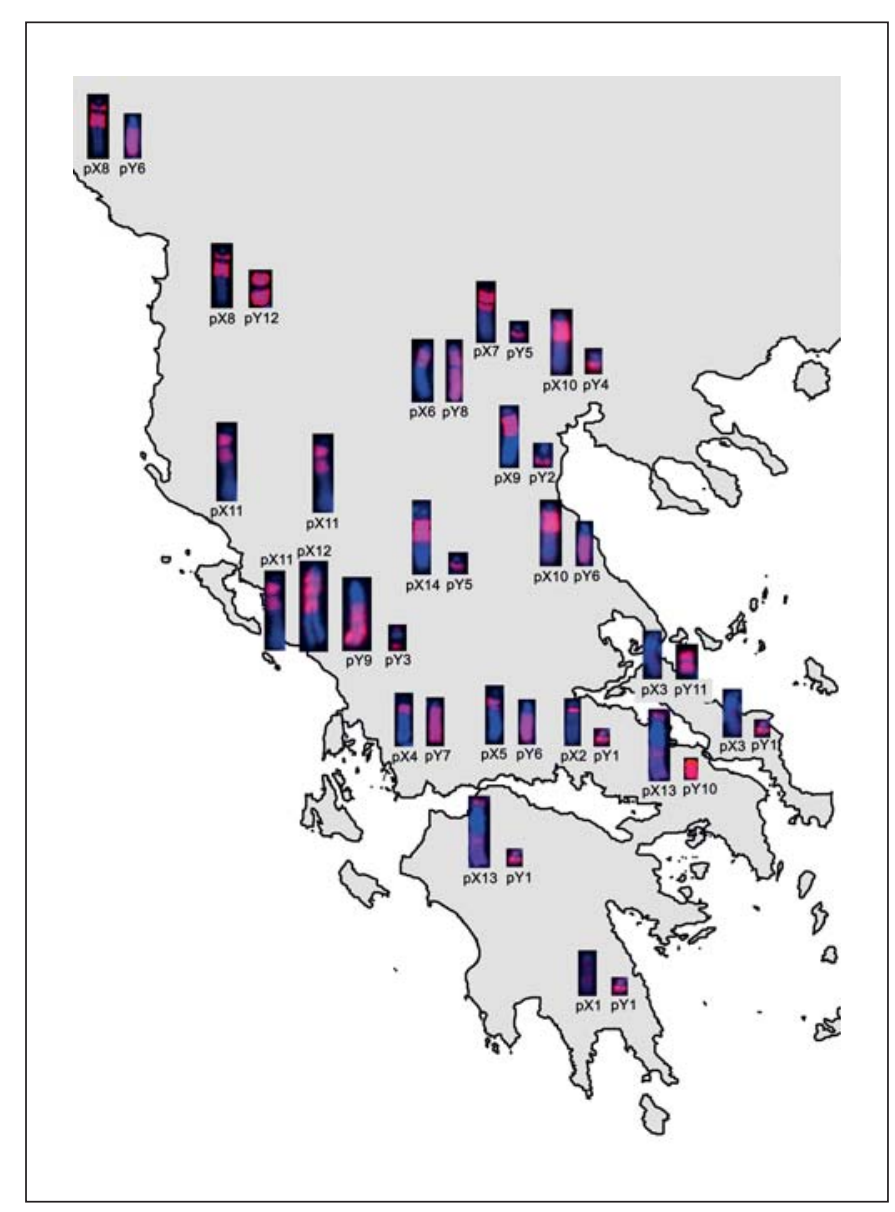

Fig. 4. Geographic distribution of the different variants of both sex chromosomes showing the painting pattern with the probe $\mathrm{Y}_{\mathrm{m} 1}$.

According to this scenario, the derived $\mathrm{X}$ chromosomes evolved via incorporation of the heterochromatic bands. The variant $\mathrm{pX} 2\left(\mathrm{X}_{1}\right)$ was the first that incorporated a heterochromatic band which is stained with the $\mathrm{Y}_{\mathrm{m} 1}$ probe. We can suppose that the heterochromatic regions or bands of the other acrocentric X chromosomes (variants pX4-pX12) originated by successive amplification or duplication of the same heterochromatic band, probably accompanied in some cases by paracentromeric inversions which could give rise to the interstitial bands that are not stained with this probe. However, the heterochromatin of the $\mathrm{X}$ chromosome variant $\mathrm{pX} 3\left(\mathrm{X}_{2}\right)$ may have a different origin as its heterochromatic block has a different composition as demonstrated by the lack of the $\mathrm{Y}_{\mathrm{m} 1}$ FISH signal. It is interesting that chromosomes previously assigned to 3 categories by C-banding, $\mathrm{X}_{2}-\mathrm{X}_{4}$ included, are in fact 9 variants according to their molecular composition ( 2 variants for $\mathrm{X}_{2}, 5$ for $\mathrm{X}_{3}$, and 2 for $\mathrm{X}_{4}$ ). 
Morphologically, the very similar submetacentric X chromosomes can be clearly differentiated by the painting pattern to 2 variants (pX13 and pX14) with independent origin. The main differences are related to the staining of the small arm and to the heterochromatic block of the long arm. While in the variant pX14 the small arm is slightly stained with the 3 painting probes, there is a subtelomeric band clearly stained in the variant pX13. Also, the heterochromatic region of pX14 is strongly stained with the $\mathrm{Y}_{\mathrm{m} 1}$ probe, while this region is not stained in pX13. The pX13 chromosome probably arose from pX3 by enlargement of the heterochromatic region of the long arm and by acquisition of a larger short arm. However, pX14 could be derived from the variants $\mathrm{pX} 9$ or $\mathrm{pX} 10$ by enlargement of the short arm. The close geographic distribution of $\mathrm{pX} 3$ with $\mathrm{pX} 13$ and $\mathrm{pX} 9$ or pX10 with pX14 is in agreement with the proposed hypothesis for the origin of $\mathrm{pX} 13$ and $\mathrm{pX} 14$ (Fig. 4). In both cases, the short arm did not originate by a pericentric inversion as was previously supposed [Giagia-Athanasopoulou and Stamatopoulos, 1997] but probably by amplification of different sequences on the original short arms. Previous results demonstrated that the repetitive DNA sequence MthAlu900 is amplified in the heterochromatic long arm but not in the heterochromatin of the short arm of $\mathrm{X}_{\mathrm{st} 1}$ [Acosta et al., 2009].

Heterochromatin Enlargement of Sex Chromosomes in Microtus

Under the assumption that the smaller acrocentric $\mathrm{X}$ and Y chromosomes of $M$. thomasi represent the ancestral sex chromosomes [Giagia-Athanasopoulou and Stamatopoulos, 1997], 2 major processes would lead to the polymorphisms: a) change in morphology from acrocentric to submetacentric or metacentric, and b) increase in size due to accumulation of different repeated DNA sequences that generated heterochromatic blocks. In addition, some molecular processes permitting the homogenization of most of the heterochromatic blocks of both sex chromosomes would have to be assumed.

Both sex chromosomes in Microtus are able to rapidly accumulate large blocks of constitutive heterochromatin, giving rise to a notable increase in sex chromosome size and leading in some species to the giant sex chromosomes, which present big heterochromatin blocks [Burgos et al., 1990; Modi, 1993; Singh et al., 2000; Marchal et al., 2004a]. In the giant sex chromosomes accumulation of heterochromatin occurs in parallel in both $\mathrm{X}$ and $\mathrm{Y}$ chromosomes, as it has occurred in the different $\mathrm{X}$ and $\mathrm{Y}$ variants described in M. thomasi and M. atticus. How-

Sex Chromosome Polymorphism of M. thomasi/M. atticus ever, the origin, composition, and evolution of these blocks are very different between species and even between both sex chromosomes of the same species. In fact, while the heterochromatic blocks of the $\mathrm{X}$ and $\mathrm{Y}$ chromosome of $M$. agrestis shared the same sequences, the heterochromatic blocks of the sex chromosomes in M. cabrerae have a different composition [Kalscheuer et al., 1996; Marchal et al., 2004a]. The sex heterochromatin in the Microtus species is highly heterogeneous within species (with different composition of the $\mathrm{X}$ and $\mathrm{Y}$ heterochromatic regions in M. cabrerae) and between species (as the composition of $M$. agrestis and M. cabrerae sex heterochromatin is different) [Marchal et al., 2004a]. The accumulation of heterochromatin in M. thomasi and M. atticus is similar to M. agrestis, where both sex chromosomes share repetitive DNA sequences despite their asynaptic condition [Singh et al., 2000; Rovatsos and Giagia-Athanasopoulou, 2012]. Unequal non-sister chromatid exchanges facilitated by repetitive DNA sequences between the heterochromatic blocks could explain the homogeneity observed in both sex chromosomes of $M$. agrestis [Singh et al., 2000], M. thomasi, and M. atticus.

The genus Microtus is one of the most karyologically variable rodent groups [Maruyama and Imai, 1981], and sex chromosome variation or polymorphism has been reported in several species. For example, 2 medium-sized $\mathrm{X}$ chromosome variants (metacentric and acrocentric) and a small Y chromosome have been described in $M$. savii, while the closely related $M$. brachycercus has a large submetacentric $\mathrm{X}$ and a large acrocentric $\mathrm{Y}$ chromosome [Galleni et al., 1994; Castiglia et al., 2008]. In M. guentheri, $3 \mathrm{X}$ chromosome variants (acrocentric, submetacentric, and metacentric) and $2 \mathrm{Y}$ chromosome variants (small- and medium-sized) have been described [Chassovnikarova et al., 2008; Mitsainas et al., 2010; Zima et al., 2013]. M. rossiaemeridionalis has 2 different $\mathrm{X}$ chromosomes varying in the size of the heterochromatic block [Fredga et al., 1990]. M. oregoni presented differences between 2 populations in both sex chromosomes due to size variation in G-banding regions and in blocks of C-banding constitutive heterochromatin [Libbus and Johnson, 1988]. Also, in M. cabrerae polymorphism affecting the giant sex chromosomes with $3 \mathrm{X}$ and $5 \mathrm{Y}$ chromosome variants has been described due to deletion of the heterochromatic blocks [Burgos et al., 1988b]. The sex chromosome polymorphism described here is the largest known up to date in microtids and probably in mammalian species.

The origin and maintaining of this polymorphism could be related to a very limited spatial mobility of sub-

Cytogenet Genome Res 2017;151:198-207 DOI: $10.1159 / 000477114$ 
terraneous $M$. thomasi and $M$. atticus combined with the rough, mountainous terrain of the Balkan Peninsula. It seems likely that inbreeding and genetic drift in the small and fragmented populations of these 2 species led to the fixation of different sex chromosomes in different regions. In fact, different sex chromosome variants are present in different regions of the distribution area of this species (Fig. 4). It has been suggested that the current geographical distribution of $M$. thomasi and $M$. atticus is a result of a northward colonization from the glacial refugee in southern Greece probably after the last glacial period (10.000-15.000 years ago) [Mitsainas et al., 2009; Rovatsos et al., 2011a]. Hence, this big sex chromosome polymorphism evolved very rapidly from the 'thomasi' chromosomal race through gradual accumulation of heterochromatin in the sex chromosomes in the northward colonization of the species, with the least amount of heterochromatin in the sex chromosomes distributed in the south-east [Mitsainas et al., 2009] (Fig. 4).

In this context, it is possible to imagine a scenario in which some isolated populations could reach enough genetic diversity to become an incipient new species, as probably has occurred previously with $M$. atticus [Rovatsos and Giagia-Athanasopoulou, 2012]. This new species could present one of the existing combinations of sex chromosome variants. If one or both sex chromosomes of the new species are the biggest ones from the existing polymorphism, it could lead into a new species with giant sex chromosomes. In fact, in one of the analyzed individuals (R18; Fig. 1e) we calculated that the X chromosome (pX12) and the Y chromosome (pY9) represent approximately $12 \%$ and $11 \%$ of the haploid genome. The giant sex chromosomes in M. cabrerae, M. chrotorrhinus, and $M$. agrestis account for a significant percentage of their genome [Modi, 1987b; Nanda et al., 1988; Kalscheuer et al., 1996; Marchal et al., 2004a], and the giant X chromosomes of $M$. agrestis and M. cabrerae represent nearly $20 \%$ and $15 \%$ of the genome, respectively [Nanda et al., 1988; Kalscheuer et al., 1996]. Our results on M. thomasi and $M$. atticus could help to underline one of the possible origins of giant sex chromosomes. We can suppose that probably a similar polymorphism variation on the sex chromosomes and processes as genetic drift fixation occurred in the origin of some of the actual species with giant sex chromosomes.

\section{Acknowledgements}

We thank Lukáš Kratochvíl for critical reading of the manuscript and comments and suggestions. This work was supported by the Spanish Ministerio de Educación y Ciencia through the project with reference CGL2009-07754 (co-funded by the European Regional Development Fund) and by the Junta de Andalucía through the programme 'Ayudas a grupos de investigación', group number BIO 220. M.R. was partially supported by Charles University Grant Agency project PRIMUS/SCI/46.

\section{Statement of Ethics}

The authors have no ethical conflicts to disclose.

\section{Disclosure Statement}

The authors have no conflicts of interest to declare.

\section{References}

Acosta MJ, Marchal JA, Fernández-Espartero C, Bullejos M, Sánchez A: Retroelements (Lines and Sines) in vole genomes: differential distribution in the constitutive heterochromatin. Chromosome Res 16:949-959 (2008).

Acosta MJ, Marchal JA, Mitsainas GP, Rovatsos MT, Fernández-Espartero $\mathrm{CH}$, et al: A new pericentromeric repeated DNA sequence in Microtus thomasi. Cytogenet Genome Res 124:27-36 (2009).

Acosta MJ, Marchal JA, Fernández-Espartero C; Romero-Fernández I, et al: Characterization of the satellite DNA Msat-160 from species of Terricola (Microtus) and Arvicola (Rodentia, Arvicolinae). Genetica 138: 1085-1098 (2010).

Acosta MJ, Romero-Fernández I, Sánchez A, Marchal JA: Comparative analysis by chro- mosome painting of the sex chromosomes in arvicolid rodents. Cytogenet Genome Res 132:47-54 (2011).

Burgos M, Jiménez R, Díaz de la Guardia R: Comparative study of G- and C-banded chromosomes of five species of Microtidae. A chromosomal evolution analysis. Genome 30: 540-546 (1988a).

Burgos M, Jiménez R, Olmos DM, Díaz de la Guardia R: Heterogeneous heterochromatin and size variation in the sex chromosomes of Microtus cabrerae. Cytogenet Cell Genet 47: 75-79 (1988b).

Burgos M, Olmos DM, Jiménez R, Sánchez A, Díaz de la Guardia R: Fluorescence banding in four species of Microtidae: an analysis of the evolutive changes of the constitutive heterochromatin. Genetica 81:11-16 (1990).
Castiglia R, Annesi F, Aloise G, Amori G: Systematics of the Microtus savii complex (Rodentia, Cricetidae) via mitochondrial DNA analyses: paraphyly and pattern of sex chromosome evolution. Mol Phylogenet Evol 46:11571164 (2008).

Chassovnikarova TG, Markov GG, Atanassov NI, Dimitrov HA: Sex chromosome polymorphism in Bulgarian populations of Microtus guentheri (Danford \& Alston, 1880). J Nat Hist 42:261-267 (2008).

Conroy CJ, Cook JA: mtDNA evidence for repeated pulses of speciation within arvicoline and murid rodents. J Mammal Evol 6:221-245 (1999).

Fredga K, Jaarola M, Ims RA, Steen H, Yoccoz NG: The 'commonvole' in Svalbard identified as Microtus epiroticus by chromosome analysis. Polar Res 8:283-290 (1990). 
Galleni L, Tellini A, Stanyon R, Cicalo A, Santini $\mathrm{L}$ : Taxonomy of Microtus savii (Rodentia, Arvicolidae) in Italy: cytogenetic and hybridization data. J Mammal 75:1040-1044 (1994).

Giagia EB: Karyotypes of '44-chromosomes' Pitymys species (Rodentia, Mammalia) and their distribution in southern Greece. Säug Mitt 32: 169-173 (1985).

Giagia EB, Ondrias JC: Karyological analysis of the vole Pitymys atticus (Rodentia, Mammalia) from Greece. Biol Gallo-Hellen 4:205-212 (1973).

Giagia-Athanasopoulou EB, Chondropoulos BP, Fraguedakis-Tsolis SE: Robertsonian chromosomal variation in the subalpine voles $\mathrm{Mi}$ crotus (Terricola) (Rodentia, Arvicolidae) from Greece. Acta Theriol 40:139-143 (1995).

Giagia-Athanasopoulou EB, Stamatopoulos C: Geographical distribution and interpopulation variation in the karyotypes of Microtus (Terricola) thomasi (Rodentia, Arvicolidae) in Greece. Caryologia 50:303-315 (1997).

Golenishchev FN, Malikov VG: The 'developmental conduit' of the tribe Microtini (Rodentia, Arvicolinae): systematic and evolutionary aspects. Russian J Theriol 5:19-26 (2006).

Hsu TC, Patton JL: Bone marrow preparations for chromosome studies, in Benirschke K (ed): Comparative Mammalian Cytogenetics, pp 454-460 (Springer, New York 1969).

Kalscheuer V, Singh AP, Nanda I, Sperling K, Neitzel H: Evolution of the gonosomal heterochromatin of Microtus agrestis: rapid amplification of a large, multimeric, repeat unit containing a 3.0-kb (GATA)11-positive, middle repetitive element. Cytogenet Cell Genet 73:171-178 (1996).

Lemskaya NA, Romanenko SA, Golenishchev FN, Rubtsova NV, Sablina OV, et al: Chromosomal evolution of Arvicolinae (Cricetidae, Rodentia). III. Karyotype relationships of ten Microtus species. Chromosome Res 18:459471 (2010).

Libbus BL, Johnson LA: The creeping vole, Microtus oregoni: karyotype and sex-chromosome differences between two geographical populations. Cytogenet Cell Genet 47:181-184 (1988).

Marchal JA, Acosta MJ, Bullejos M, Díaz de la Guardia R, Sánchez A: Sex chromosomes, sex determination, and sex-linked sequences in Microtidae. Cytogenet Genome Res 101:266273 (2003).
Marchal JA, Acosta MJ, Nietzel H, Sperling K, Bullejos $\mathrm{M}$, et al: $\mathrm{X}$ chromosome painting in Microtus: origin and evolution of the giant sex chromosomes. Chromosome Res 12:767-776 (2004a).

Marchal JA, Acosta MJ, Bullejos M, Díaz de la Guardia R, Sánchez A: A repeat DNA sequence from the $\mathrm{Y}$ chromosome in species of the genus Microtus. Chromosome Res 12: 757-765 (2004b).

Marchal JA, Acosta MJ, Bullejos M, Puerma E, Díaz de la Guardia R, Sánchez A: Distribution of 11-retroposons on the giant sex chromosomes of Microtus cabrerae (Arvicolidae, Rodentia): functional and evolutionary implications. Chromosome Res 14:177-186 (2006).

Marchal JA, Acosta MJ, Bullejos M, Diaz de la Guardia R, Sanchez A: Origin and spread of the $S R Y$ gene on the $\mathrm{X}$ and $\mathrm{Y}$ chromosomes of the rodent Microtus cabrerae : role of L1 elements. Genomics 91:142-151 (2008).

Maruyama T, Imai HT: Evolutionary rate of the mammalian karyotype. J Theor Biol 90:111121 (1981).

Mitsainas GP, Rovatsos MT, Rizou EI, GiagiaAthanasopoulou EB: Sex chromosome variability outlines the pathway to the chromosomal evolution in Microtus thomasi (Rodentia, Arvicolinae)'. Biol J Linn Soc 96:685-695 (2009).

Mitsainas GP, Rovatsos MT, Giagia-Athanasopoulou EB: Heterochromatin study and geographical distribution of Microtus species (Rodentia, Arvicolinae) from Greece. Mammal Biol 75:261-269 (2010).

Modi WS: Phylogenetic analyses of chromosomal banding patterns among the nearctic Arvicolidae (Mammalia: Rodentia). Syst Zool 36: 109-136 (1987a).

Modi WS: C-banding analysis and the evolution of heterochromatin among arvicolid rodent. J Mammal 68:704-714 (1987b).

Modi WS: Heterogeneity in the concerted evolution process of a tandem satellite array in meadow mice (Microtus). J Mol Evol 37:4856 (1993).

Musser GG, Carleton MD: Superfamily Muroidea, in Wilson DE, Reeder DM (Eds) Mammal Species of the World: A Taxonomic and Geographic Reference, pp 894-1531 (Johns Hopkins University Press, Baltimore 2005).
Nanda I, Neitzel H, Sperling K, Studer R, Epplen JT: Simple GATCA repeats characterize the $\mathrm{X}$ chromosome heterochromatin in Microtus agrestis, European field vole (Rodentia, Cricetidae). Chromosoma 96:213-219 (1988).

Rovatsos MT, Giagia-Athanasopoulou EB: Taxonomical status and phylogenetic relations between the 'thomasi' and 'atticus' chromosomal races of the underground vole Microtus thomasi (Rodentia, Arvicolinae). Mamm Biol 77:6-12 (2012).

Rovatsos MT, Mitsainas GP, Paspali G, Oruci S, Giagia-Athanasopoulou EB: Geographical distribution and chromosomal study of the underground vole Microtus thomasi in Albania and Montenegro. Mamm Biol 76:22-27 (2011a).

Rovatsos MT, Marchal JA, Romero-Fernández I, Fernández FJ, Giagia-Athanosopoulou EB, Sánchez A: Rapid, independent, and extensive amplification of telomeric repeats in pericentromeric regions in karyotypes of arvicoline rodents. Chromosome Res 19:869-882 (2011b).

Rovatsos MT, Marchal JA, Romero-Fernández I, Cano-Linares M, Fernández FJ, et al: Molecular and physical characterization of the complex pericentromeric heterochromatin of the vole species Microtus thomasi. Cytogenet Gen Res 144:131-141 (2014).

Singh A, Henschel S, Sperling K, Kalscheuer V, Neitzel H: Differences in the meiotic pairing behavior of gonosomal heterochromatin between female and male Microtus agrestis: implications for the mechanism of heterochromatin amplification on the $\mathrm{X}$ and $\mathrm{Y}$. Cytogenet Cell Genet 91:253-260 (2000).

Sitnikova NA, Romanenko SA, O’Brien PC, Perelman PL, Fu B, et al: Chromosomal evolution of Arvicolinae (Cricetidae, Rodentia). I. The genome homology of tundra vole, field vole, mouse and golden hamster revealed by comparative chromosome painting. Chromosome Res 15:447-456 (2007).

Volobouev VT, Gallardo MH, Graphodatsky AS: Rodents cytogenetics, in:O'Brien SJ, Nash WG, Menninger JC (eds): Atlas of Mammalian Karyotypes, pp 173-176 (Wiley, Chichester 2006).

Zima J, Arslan A, Benda P, Macholán M, Kryštufek B: Chromosomal variation in social voles: a Robertsonian fusion in Günther's vole. Acta Theriol 58:255-265 (2013).
Sex Chromosome Polymorphism of M. thomasi/M. atticus
Cytogenet Genome Res 2017;151:198-207 DOI: $10.1159 / 000477114$ 